\title{
أهم رسائل المؤتمر الدولي لمعهد التخطيط القومي "الطاقة والتنمية المستدمة"
}

\section{القاهرة: 21-22 نوفمبر 2020}

* أ.د. نيفين كمال

عقد معهد التخطيط القومي مؤتمره الدولي السنوي تحت عنوان " الطاقة والتتمية المستدامة" برئاسة أ. د. علاء زهران رئيس المعهد ورئيس المؤتمر، وقد حضر الجلسة الافتتاحية كل من أ.د. أحمد كمالي نائباً عن معالي أ.د. هالة السعيد وزيرة التخطيط والتمية الاقتصادية ورئيس مجلس إدارة المعهد، ومعالي السيد الدكتور/ محمد شاكر وزير الكهرباء والطاقة المتجددة، ومعالي السيد المهندس/طارق الملا وزير البترول والثروة المعدنية. وتتاول المؤتمر عبر جلساته الست على مدار يومين، قضايا استدامة الطاقة والتتمية من واقع خبرات دولية وإقليمية، والطاقة في إطار المتغيرات العالمية والإقليمية، ومستقبل الطاقة المتجددة عالمياً وإقليمياً، ونظم الطاقة المستدامة، وقضايا الطاقة وتغير المناخ، وتخطيط الطاقة في مصر • وقد شارك في المؤتمر عدة جهات دولية وإقليمية ومحلية تعمل في مجالات الطاقة والتنمية المستدامة، وهى: برنامج الأمم المتحدة الإنمائي (UNDP)، ولجنة الأمم المتحدة الاقتصادية والاجتماعية لغرب آسيا (ESCWA)، والوكالة الدولية للطاقة المتجدة (IRENA)، والمجلس العالمي للطاقة (WEC)، والمعهد الدولي لتحليل النظم التطبيقية (IIASA)، ومنظمة الأقطار العربية المصدرة للبترول (OAPEC)، بالإضافة إلى الجهات المحلية المتمثلة في هيئة الطاقة الجديدة والمتجددة، وجهاز شؤون البيئة، ومرفق تتظيم أنشطة سوق الغاز ، والشركة القابضة لكهرباء مصر ، والشركة المصرية لنقل الكهرباء، وعدد من الخبراء والباحثين. وخلصت الأوراق المقدمة والمناقشات التي دارت على مدار اليومين إلى مجموعة من الرسائل، أهم هذه الرسائل ما يلي: - ماني:

\section{1}

عدم توافر مصادر من الطاقة التقليدية تكفي سكان العالم وفقاً لأنماط الاستهلاك الحالية، مما يتطلب التكامل بين مصادر الطاقة المختلفة، التقليدية والمتجددة، وكذلك التكامل بين الاستراتيجيات القطاعية، والتعاون والتنسيق بين جميع الأطراف ذات الصلة، سواء حكومية أو قطاع خاص، أو محلية أو إقليمية أو عالمية. اتباع التفكير المنظومي، واستخدام الأدوات المستحدثة في علوم المنظومات، وذلك بهدف الته التعامل السليم مع عمليات الطاقة سواء في التحليل أو التصميم أو التشغيل أو استشراف 
المستقبل. وتطبيقاً لذلك تبرز أهمية ربط قطاع الطاقة بكافة متغيرات الاقتصاد الكلي في لهي كمال إطار شامل، ودمج الابتكارات في شكل منظومي متكامل. الترابط القوي بين الطاقة والمياه، الذي يتطلب استخدام الموارد بكفاءة، وتطبيق التكنولوجيات التي تساعد على رفع كفاءة الاستخدام، والتحول إلى استخدام الطاقة

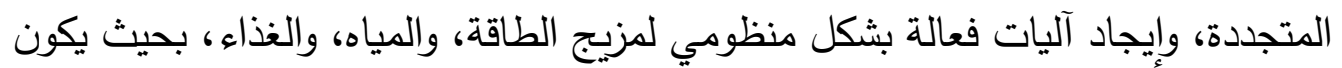
هناك إطار مؤسسي على مستوى الدولة يمكن تتفيذه في إطار زمني محدد. تتطلب الإدارة الجيدة لقطاع الطاقة في ضوء التطورات المستجدة إجراء تحسينات مستمرة في كفاءة إنتاج واستخدام الطاقة، وتطوير سوق الطاقة، وتتثيف المواطنين وزيادة الوعي،

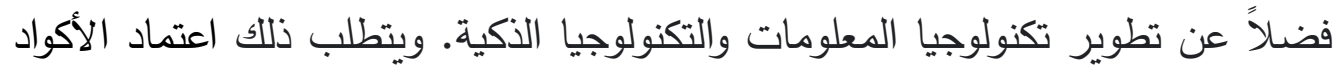
ذات الصلة وتفعيل القائم منها مثل كود تحسين كفاءة الطاقة في المباني، بالإضافة إلى تطوير تقنيات ونظم الكفاءة للتطبيقات المختلفة في المباني والنقل والصناعة.

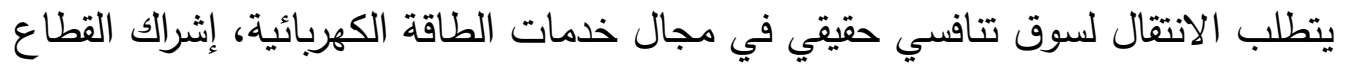

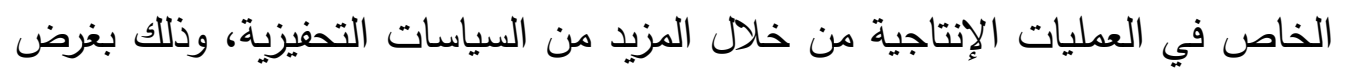
تقديم الطاقة الكهربائية بأسعار تنافسية، تعمل على دفع النمو الصناعي من ناحية، وتوفير

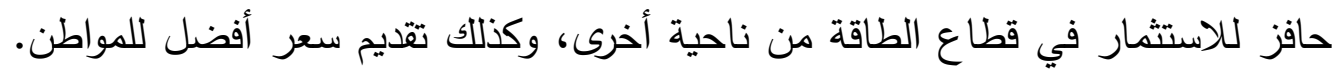

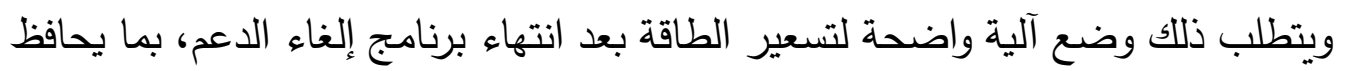

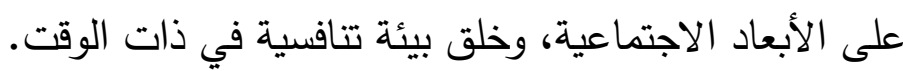
التوجه نحو توطين صناعة التقنيات والمعدات ذات الصلة بالطاقة، خاصة الطاقة المتجددة، والعمل على توسيع أسواقها، لخلق فرص عمل إضافية ومتتوعة، وزيادة العوائد الاقتصادية والاجتماعية والبيئية للتحول في نظام الطاقة المصري. وكذلك التوجه نحو

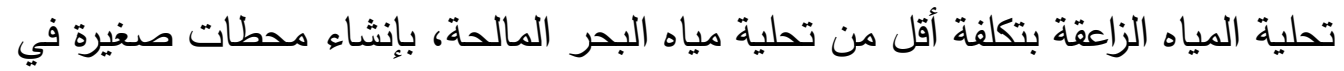
الوادي، يمكن تمويلها من كل من الحكومة، والقطاع الخاص، والصناديق السيادية، والجهات الدولية. ويُكن في هذا السياق مراجعة ودعم برامج التعاون العربي، والدولي لتعميق التصنيع المشترك. الانتقال من الحديث عن عرض الطاقة إلى مناقثة القضايا المتعلقة بالطلب على الطاقة،

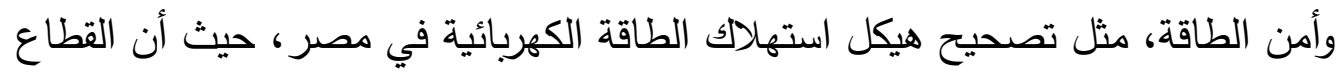

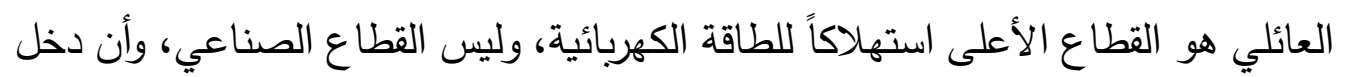

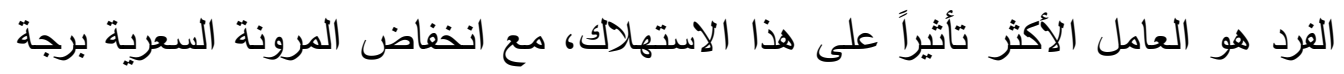

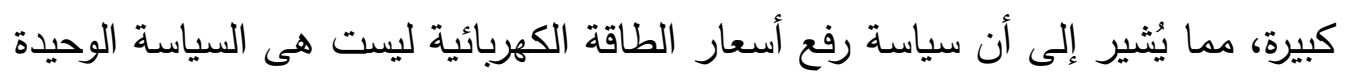
لترشيد استهلالك القطاع العائلي من الطاقة الكهربائية التهائ.

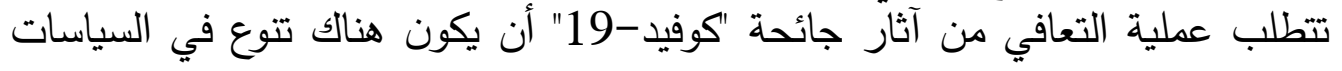

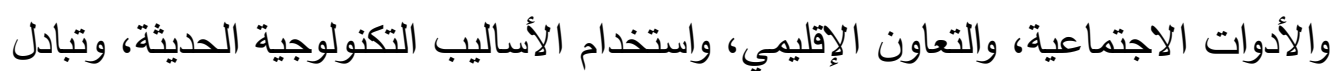

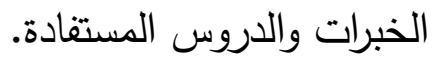




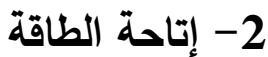

تمكنت مصر من تأمين التغذية الكهربائية لكافة القطاعات، عن طريق زيادة قدرات

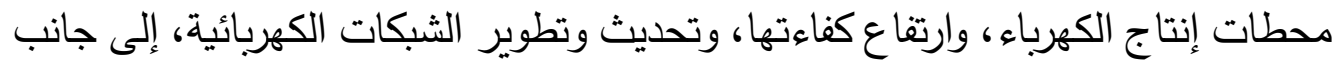

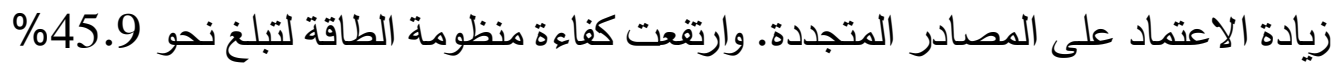
في عام 2019/2018، بسبب إنشاء الدحطات الجديدة، والصيانة، والاعتماد على الدورة

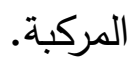

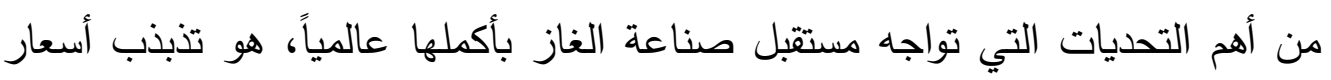

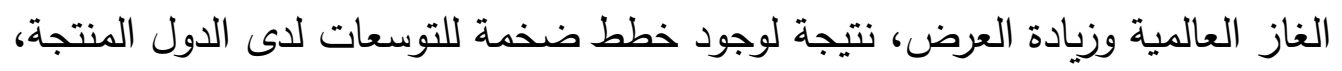

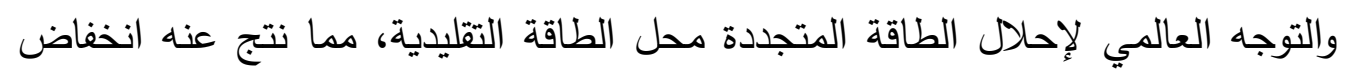

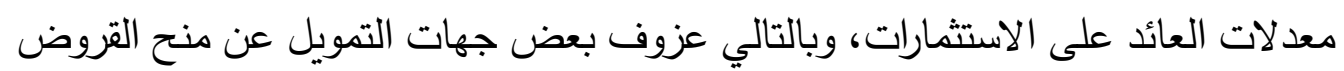
للاستثمار في أنشطة الغاز الطبيعي. تتطلب زيادة استهلاك الطاقة الكهربائية في قطاعات الاستخدام النهائي، تطوير البنية

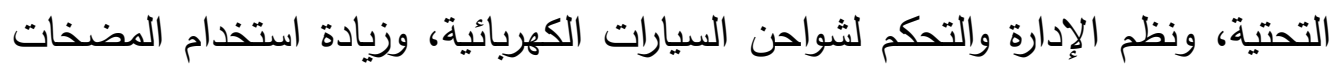

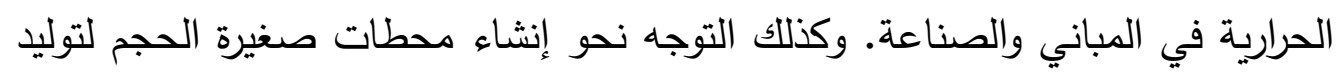

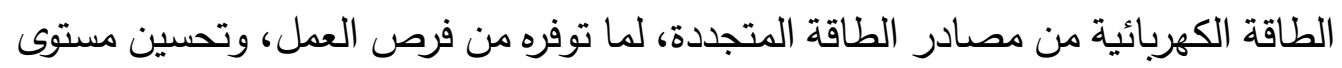
المعيثة، وتحويل المستهلك إلى منتج للطاقة، وتخفيض تكلفة البنية الأساسية، وتوطين

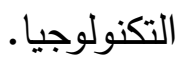

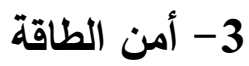

هناك معضلة ثلاثية تتعلق بكل من أمن الطاقة، والعدالة في توفير الطاقة، والاستدامة

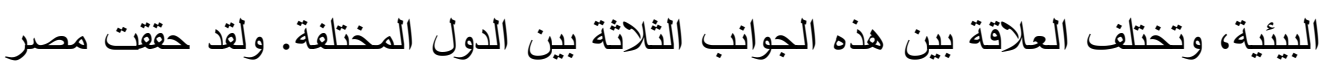
تقدماً كبيراً في مجال الطاقة خلال الفترة الماضية، مما جعلها تحقق تحسناً في مجال

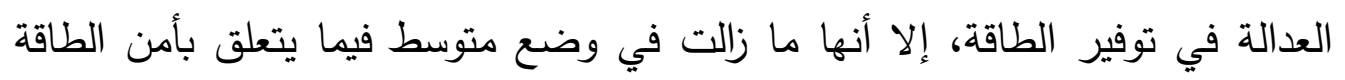

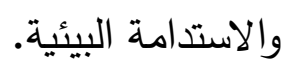

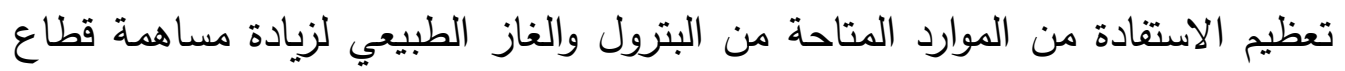

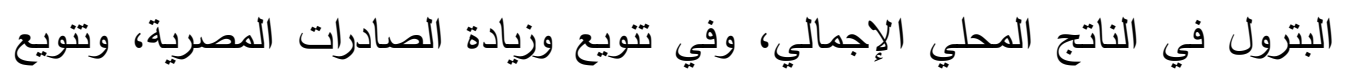

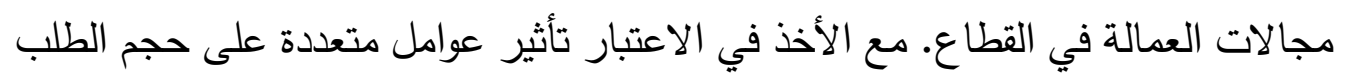
على الطاقة في الأجل الطويل، مثل ندرة المياه والغذاء.

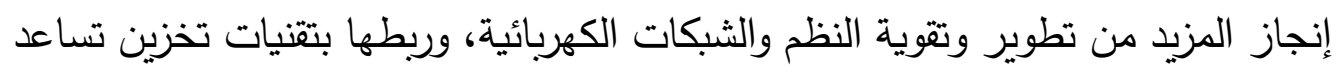
على تنظيم إمدادها بالكهرباء المنتجة من المصادر المتجددة، ورقمنة نظم التحكم ذات 
يتطلب تحويل مصر إلى مركز إقليمي لتجارة الطاقة تطوير تكنولوجيا المعلومات لحماية

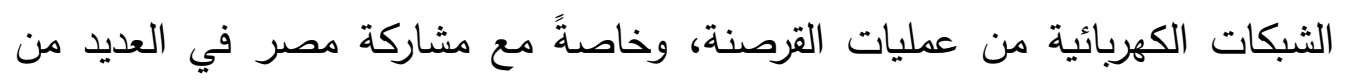

$$
\text { مشروعات الربط الكهربائي الإقليمي. }
$$

4- استدامة الطاقة وتغير المناخ النئ

أن سياسات تغير المناخ الخاصة بالهدف الثالث عشر من الأهداف الأممية للتنمية

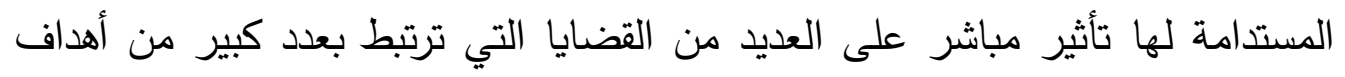

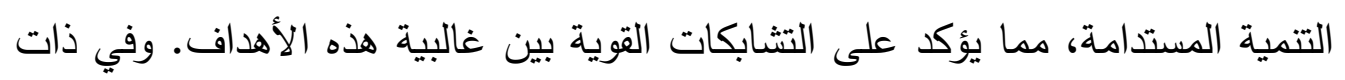

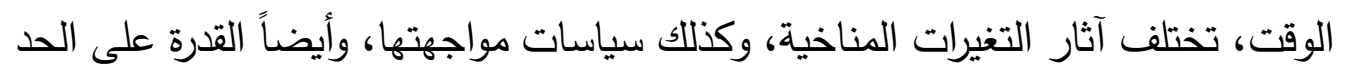
من آثارها، من دولة لأخرى. التخفيف من الآثار السلبية المتعددة لتغير المناخ يتطلب تكامل العديد من السياسات التي

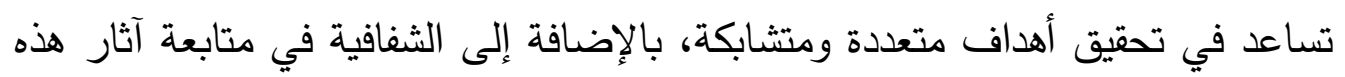

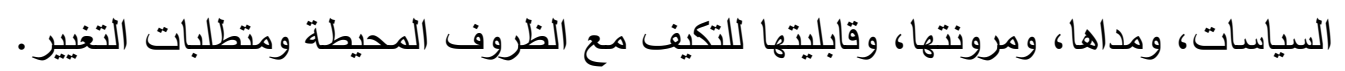
وكذلك يتطلب تضافر الجهود بين المؤسسات المختلفة على كافة الأصعدة الوطنية

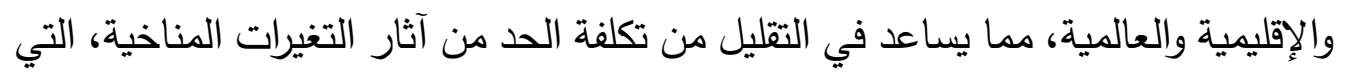
تكون أكثر تأثيراً على الأسر منخفضة مالفية الدخل. محدودية قدرة الكرة الأرضية على استيعاب انبعاثات الطاقة، يحتم التحول لاستخدام الطاقة

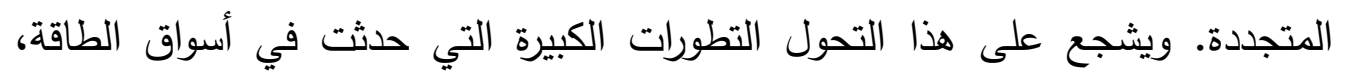
وتتاقص أسعار الطاقة المتجددة بنسبة كبيرة خلال العقد الأخير.

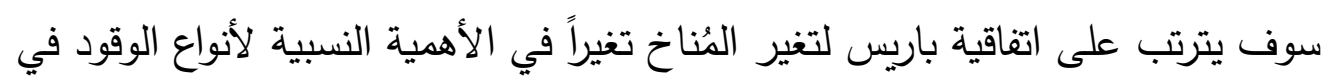

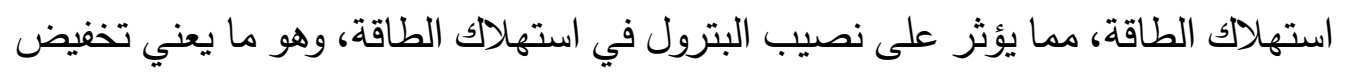

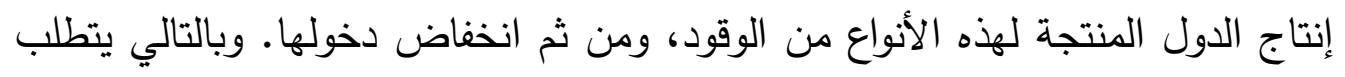

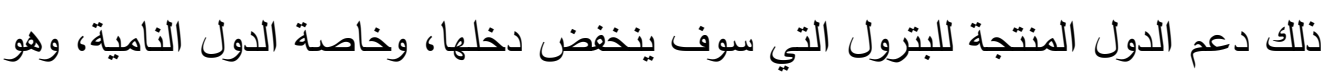
ما نصت عليه الاتفاقية. التركيز على القطاعات والتقنيات ذات التأثير الأعلى في خفض الانبعاثات التات الكربونية،

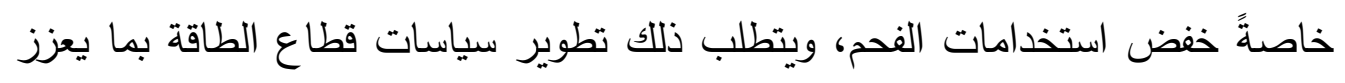

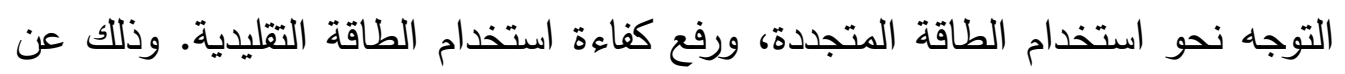

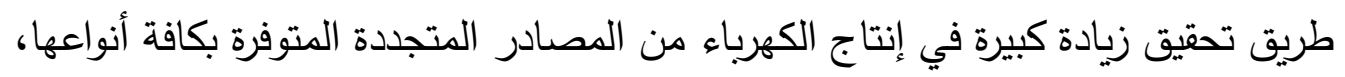

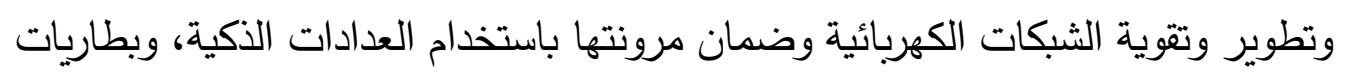

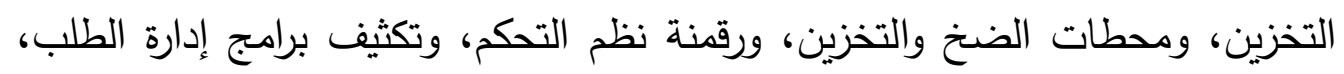

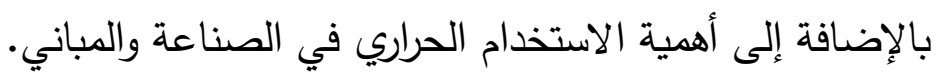


التغلب على التحديات التي تعوق تحفيز الاستثمار في الطاقة المتجددة، والمتمثلة في عدم

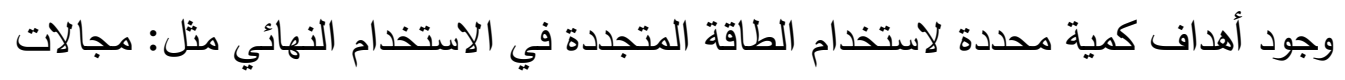

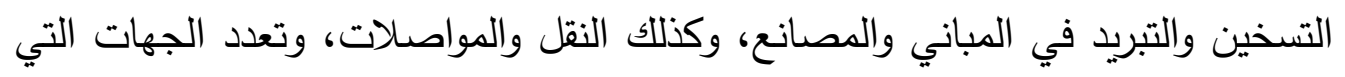

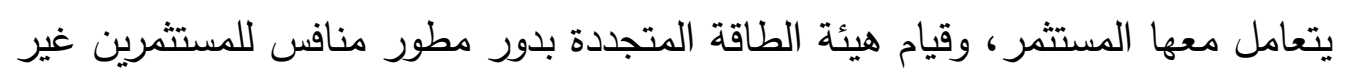

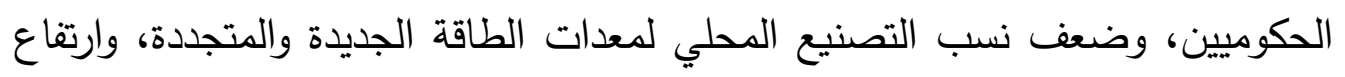
التكاليف المرتبطة باللوجستيات والنقل، وارتفاع تكلفة تمويل المشروعات الصغيرة

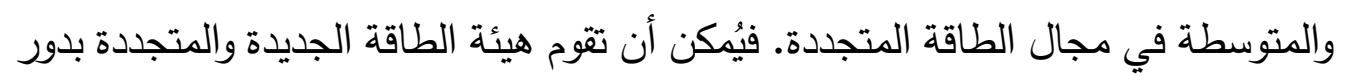

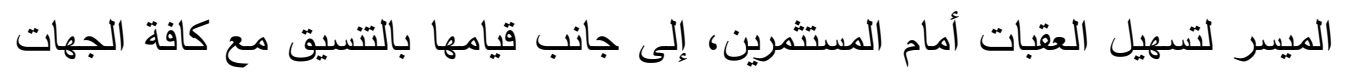
والمؤسسات الحكومية الأخرى لطرح فرص استثمارية جاهزة بكافة الإجراءات الحكومية،

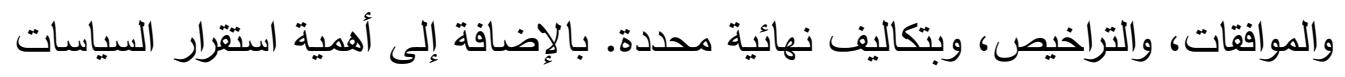

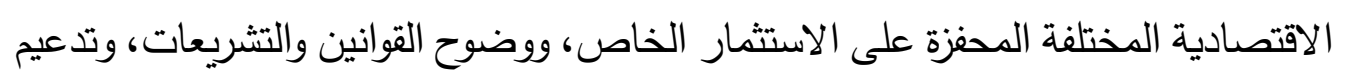
البحث العلمي والابتكار في مجال تكنولوجيات الطاقة المتجدة، وإعداد الكوادر البشرية للعمل في هذا المجال. حققت مصر تقدماً في إصدار أول طرح للسندات الخضراء ذات السيادة العامة في منطقة

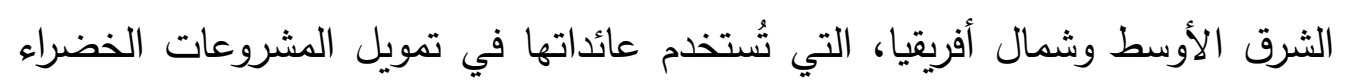

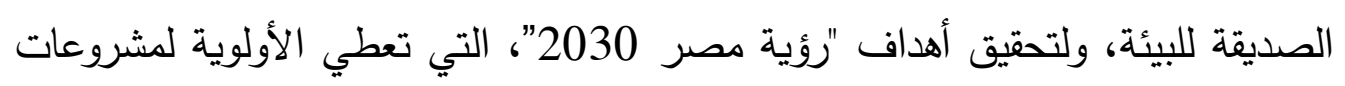
الاستثمار الأخضر. زيادة الاستثار في مجال الطاقة الشمسية كأحد مصادر توليد الطاقة الكهربائية، خاصةً

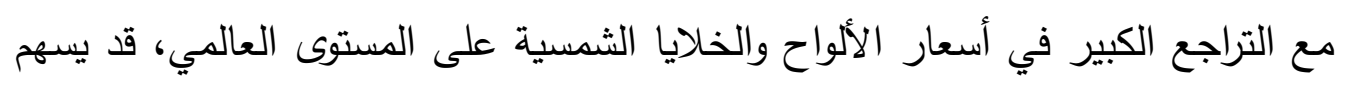
في توفير الطاقة الكهربائية بأسعار محفزة لنمو القطاع الصناعياعي مقارنةً بالأسعار الحالية.

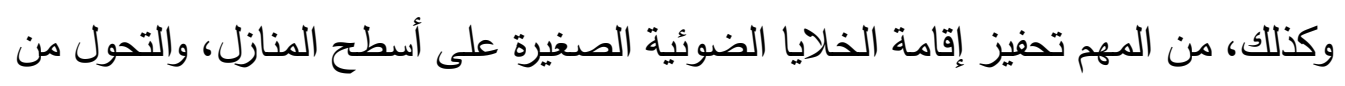

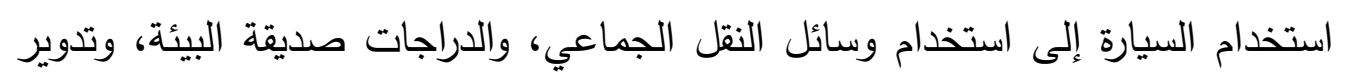

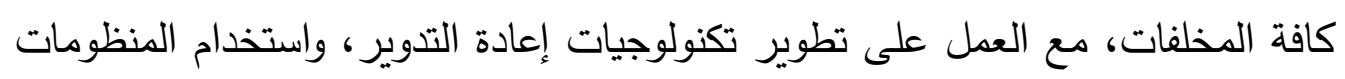

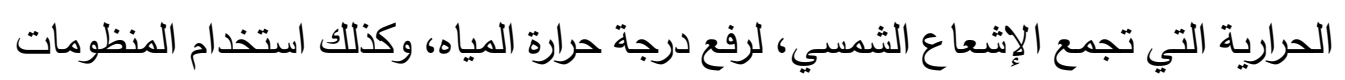
الضوئية في تحويل ضوء الشمس مباشرة إلى طاقة كهربائية.

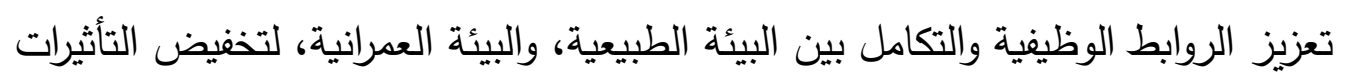

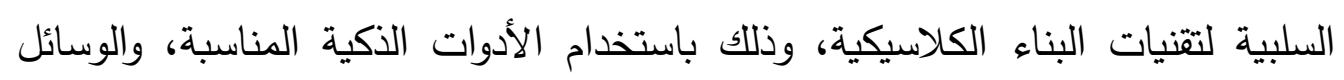

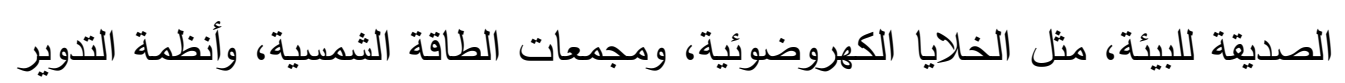

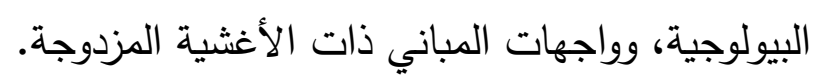


اعتماد تكنولوجيات الطاقة النظيفة، مثل تكنولوجيات تقليل حرق الغاز، وفصل الكربون

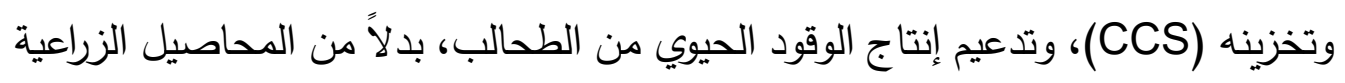
التي ارتفعت أسعارها بنسبة كبيرة، نتيجة استخدامها في إنتاج الوقود الحيوي.

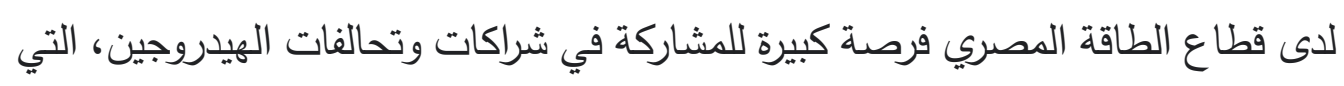
ستتيح لمصر أن تكون في طليعة مصدري الهيدروجين الأخضر بحلول نهاية عام 2050.

5- المؤسسات والتنظيم والتشربع

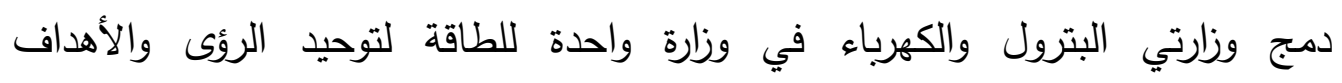

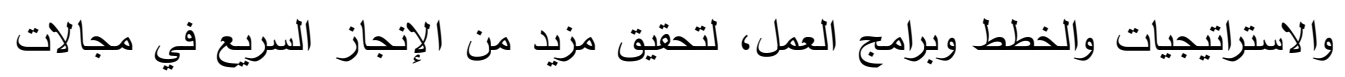

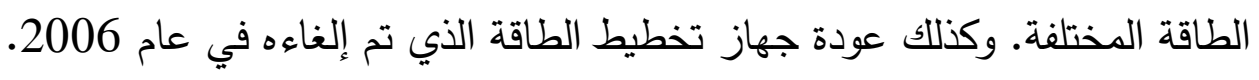

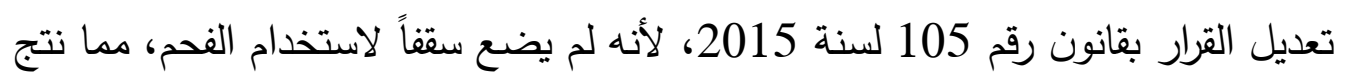

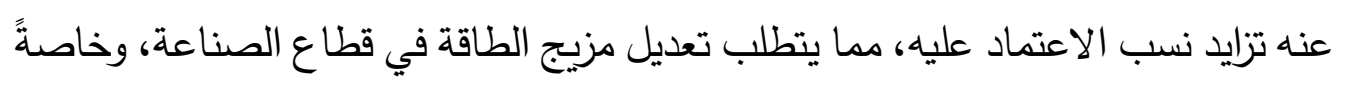

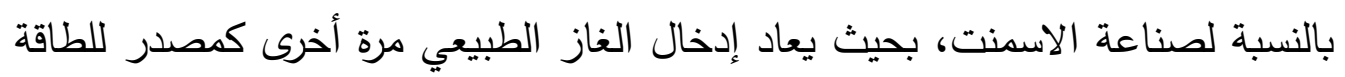
في هذه الصناعة، وأن يتم تخفيض الاعتماد على الفحم تدريجياً، حتى يتم إلغاء الاعتماد عليه نهائياً.

استكمال تعديل الإطار التظيمي لقطاع الطاقة من خلال توضيح دور كل جهة، وضمان عدم تعارض المصالح، وانسيابية الإجراءات، بالإضافة إلى استكمال الإطار التشريعي

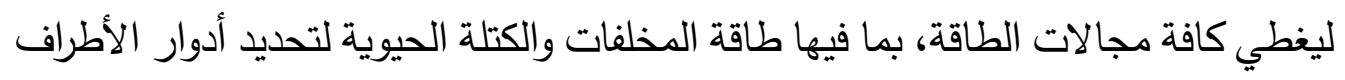

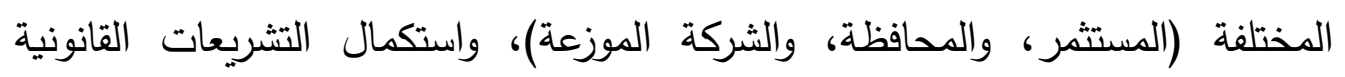

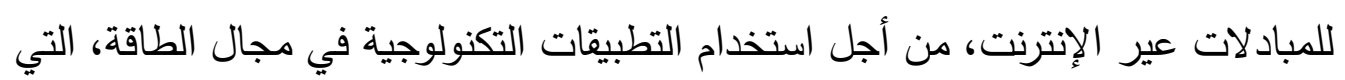
توفر في استهلاك الطاقة. بالرغم من استخدام العديد من الآليات التشريعية والتتظيمية لتحقيق تتافسية سوق الطاقة الكهربائي، إلا أن الانتقال إلى سوق تتافسي فعلي قد يستغرق المزيد من الوقت، مع إدخال

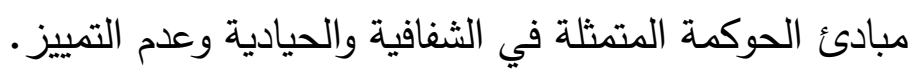

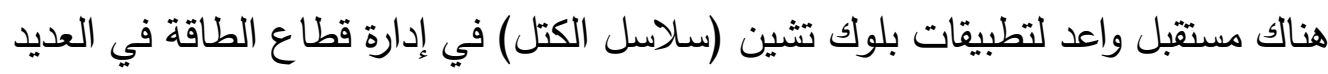
من المجالات، من أهمها مبادلات الطاقة الكهربائية من خلال العقود الذكية الإلكترونية،

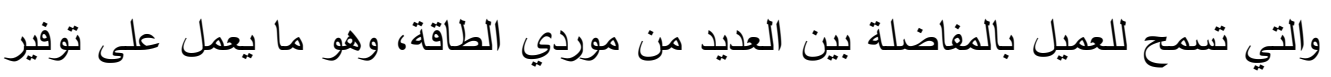
الطاقة الكهربائية بأسعار تتافسية. كما يجب استخدام تكنولوجيا البلوك تشين كأداة لتسريع تحول الطاقة. 